\title{
A Preliminary Investigation of General and Technique-specific Assessments for the Evaluation of Laparoscopic Technical Skills
}

\author{
Ashley Vergis ${ }^{1}$, Sarah Steigerwald ${ }^{1}$ \\ 1. Surgery, University of Manitoba \\ Corresponding author: Ashley Vergis, ashleyvergis@yahoo.com
}

\section{Abstract \\ Background}

Both general and technique-specific assessments of technical skill have been validated in surgical education. The purpose of this study was to assess the correlation between the objective structured assessment of technical skills (OSATS) and the global operative assessment of laparoscopic skills (GOALS) rating scales using a high-fidelity porcine laparoscopic cholecystectomy model.

\section{Methods}

Post-graduate year-one general surgery and urology residents $(n=14)$ performed a live laparoscopic porcine cholecystectomy. Trained surgeons rated their performance using OSATS and GOALS assessment scales.

\section{Results}

Pearson's correlation coefficient between OSATS and GOALS was 0.96 for overall scores. It ranged from 0.78 - 0.89 for domains that overlapped between the two scales.

\section{Conclusion}

There is a very high correlation between OSATS and GOALS. This implies that they likely measure similar constructs and that either may be used for summative-type assessments of trainee skill. However, further investigation is needed to determine if technique-specific assessments may provide more useful feedback in formative evaluation.

Received 07/17/2017

Review began 08/02/2017 Review ended 10/05/2017 Published 10/07/2017

() Copyright 2017

Vergis et al. This is an open access article distributed under the terms of the Creative Commons Attribution License CC-BY 3.0., which permits unrestricted use, distribution, and reproduction in any medium, provided the original author and source are credited.
Categories: Medical Education, General Surgery

Keywords: assessment tool, laparoscopic surgery, technical skills, medical education

\section{Introduction}

Valid and reliable methods of assessing technical performance are essential for surgical training programs and educational research [1]. They afford the maintenance of academic standards and function to provide feedback to learners as they progress through training.

Surgical skills are most commonly assessed using in-training evaluation reports (ITERs) in Canada. These are composed of rating scales designed to assess the Canadian Medical Education Directions for Specialists (CanMEDS) competencies in addition to technical skills (Figure 1) [2]. However, criticisms of using ITERs to evaluate technical skills include poor validity and limited inter and intra-rater reliability [3-5]. 


\section{Cureus}
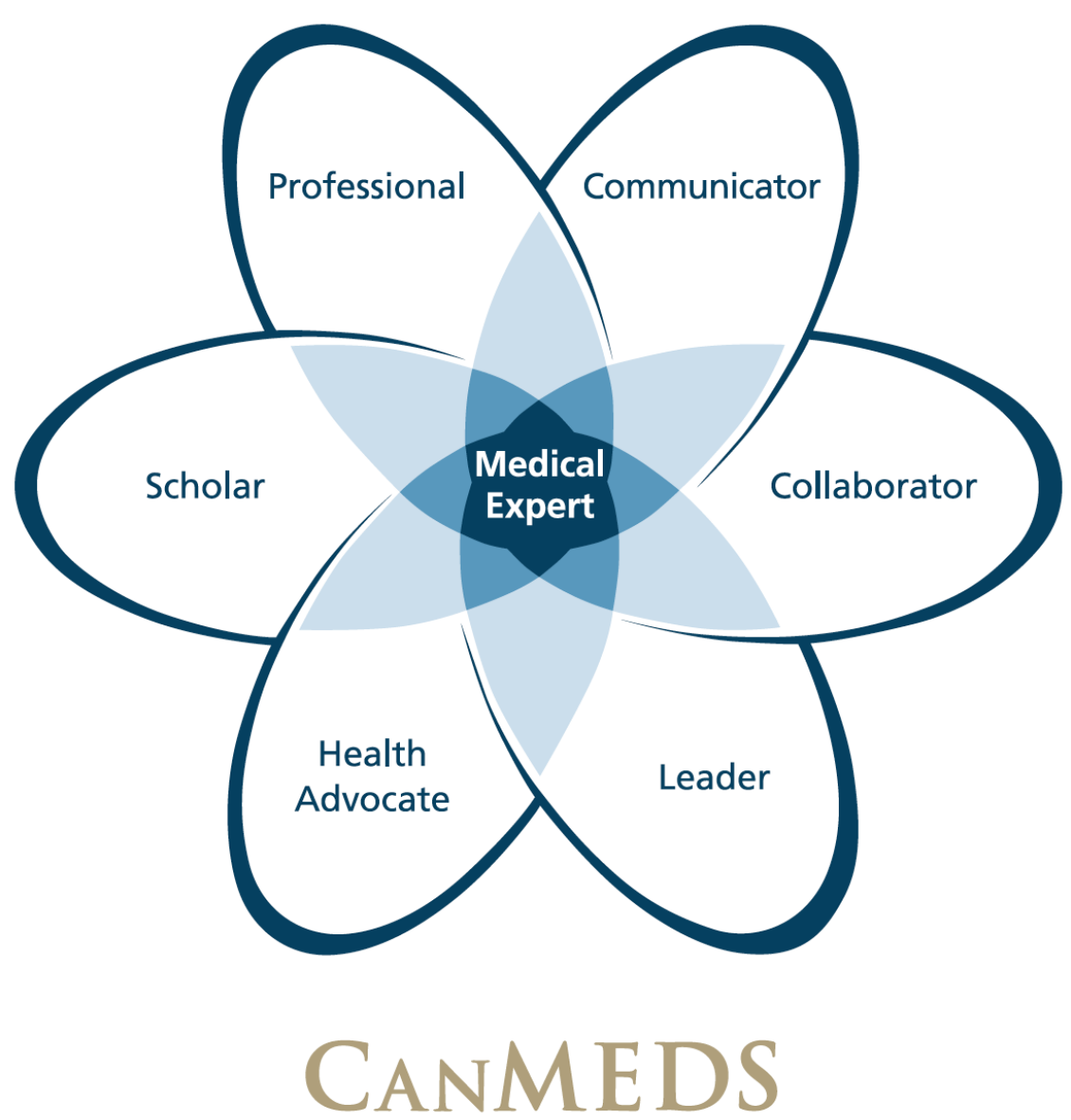

\section{FIGURE 1: CanMEDS Framework}

Copyright $\odot 2015$ The Royal College of Physicians and Surgeons of Canada. Reproduced with permission.

CanMEDS: Canadian Medical Education Directions for Specialists

In response, many methods have been developed to objectively measure technical skills. Two important methods in laparoscopic general surgery include the objective structured assessment of technical skills (OSATS) and the global operative assessment of laparoscopic skills (GOALS) global rating scales [6]. Each consists of domains that encompass aspects of operative performance (OSATS $n=7$, maximum score 35; GOALS $n=5$, maximum score 25 ) that are anchored on a 5-point Likert scale. OSATS and GOALS have been shown to be valid and reliable for bench and operative settings in multiple investigations [6-17].

OSATS was initially developed as a general performance-based, bench-model examination consisting of eight 15-minute bench-model stations but has moved from use in the laboratory setting to use in the operating theatre (Figure 2). 


\section{Cureus}
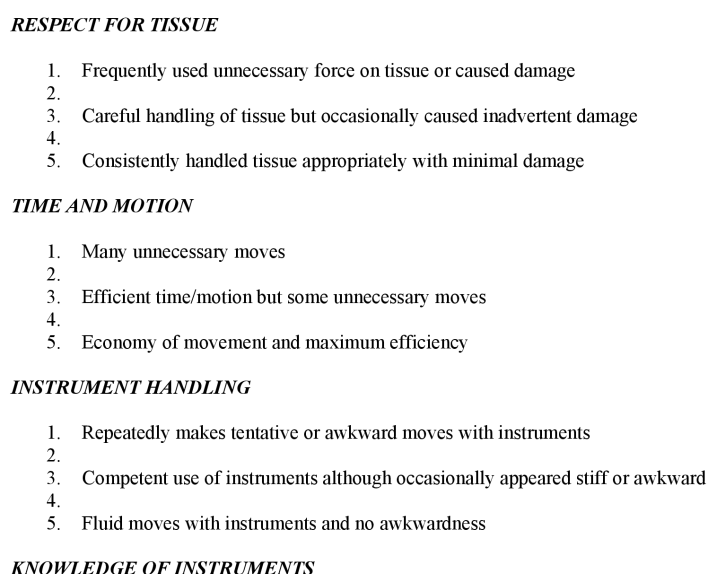

\section{KNOWLEDGE OF INSTRUMENTS}

1. Frequently asked for wrong instrument or used inappropriate instrument

3. Knew names of most instruments and used appropriate tool for task

4 .

Obviously familiar with the instruments and their names

FLOW OF PORCEDURE
1. Frequently stopped operating or needed to discuss next move
Demonstrated ability for forward planning with steady progression of operative procedure
5. Obviously planned course of operation with effortless flow from one move to the next

USE OF ASSISTANTS

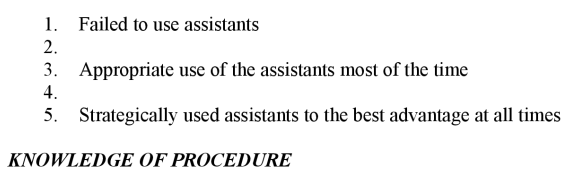

3. Appropriate use of the assistants most of the time

5. Strategically used assistants to the best advantage at all times

KNOWLEDGE OF PROCEDURE

1. Required specific instruction at most steps
3.
4. Knew all important steps of the procedure
5. Familiar with all aspects of the procedure

\section{FIGURE 2: Objective Structured Assessment of Technical Skills} (OSATS)

GOALS is a technique-specific tool developed to specifically assess operative skills in laparoscopic surgery (Figure 3). The assumption is that laparoscopic surgery requires a more specialized assessment due to the unique, technique-specific skills associated with it. These include depth perception through a limited twodimensional viewing field and bimanual dexterity with laparoscopic instruments. However, the presence of multiple, validated-forms of assessment calls into question the need for both general and technique-specific assessments of technical skills in surgical education. 


\section{DEPTH PERCEPTION}

. Constantly overshoots target, wide swings, slow to correct 2.

3. Some overshooting or missing target, but quick to correct

5. Accurately directs instruments in the correct plane to target

\section{BIMANUAL DEXTERITY}

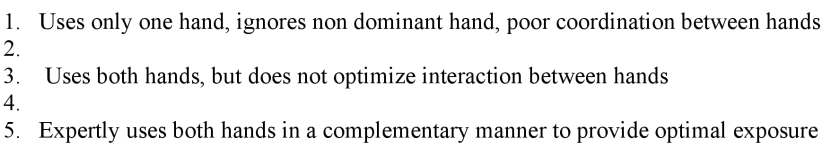

\section{EFFICIENCY}

1. Uncertain, inefficient efforts, many tentative movements, constantly changing focus or persisting without progress

3.

5. Confident, efficient and safe conduct, maintains focus on task until it is better performed by way of an alternative approach

\section{TISSUE HANDLING}

1. Rough movements, tears tissue, injures adjacent structures, poor grasper control, grasper 2. frequently slips

3. Handles tissue reasonably well, minor trauma to adjacent tissue (i.e. occasional unnecessary bleeding or slipping of the grasper)

Handles tissues well, applies appropriate traction, negligible injury to adjacent structures

\section{AUTONOMY}

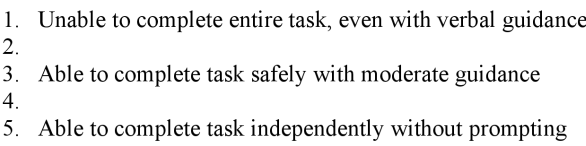

5. Able to complete task independently without prompting

\section{FIGURE 3: Global Operative Assessment of Laparoscopic Skills} (GOALS)

The purpose of this investigation is to evaluate the correlation between the OSATS and the GOALS rating scales using a live-porcine laparoscopic cholecystectomy model in a summative fashion.

\section{Materials And Methods}

Fourteen postgraduate, year-one general surgery $(n=11)$ and urology $(n=3)$ residents at the University of Manitoba participated in this study during their surgical skills sessions. This number represents all of the trainees registered in these sessions during the study period. The residents each performed a cholecystectomy using a live porcine animal model. Prior to this, they were given didactic instruction on relevant equipment setup and use, relevant anatomy, and procedural steps.

Each performance was evaluated using OSATS and GOALS global rating scales by one of two faculty evaluators. Both evaluators were academic laparoscopic surgeons with extensive experience and training in using each rating scale. Additionally, both evaluators participated in the external validation of GOALS. We were only able to have one surgeon present at each performance due to logistical constraints. Each surgeon evaluated one-half of the participants. The University of Manitoba Health Ethics Research Board granted ethical approval to carry out the study by written consent (HS13026). The University of Manitoba Bannatyne Campus Animal Protocol Managment and Review Committee approved the use of animals in this study (15020). 


\section{Cureus}

\section{Data analysis}

Correlation between the OSATS and GOALS rating scales was examined using Pearson's coefficient, IBM Statistical Package for Social Sciences (SPSS), Version 20.0 (IBM Corp., Armonk, NY). This was carried out for overall scores and overlapping domains on the two scales.

\section{Results}

Mean (overall) and overlapping individual OSATS and GOALS domains are presented in Table 1.

\begin{tabular}{|c|c|c|}
\hline & OSATS $/ 35$ & GOALS /25 \\
\hline Overall & $16.8(9-26), 48 \%(25.7 \%-74.3 \%)$ & $12.4(7-19), 49.6 \%(28 \%-76 \%)$ \\
\hline Respect for tissue/tissue handling & $2.6(1-5)$ & $2.7(1-5)$ \\
\hline Time and motion/efficiency & $2.1(1-4)$ & $2.3(1-4)$ \\
\hline Instrument Handling/Bi manual dexterity & $2.4(1-4)$ & $2.3(1-4)$ \\
\hline
\end{tabular}

\section{TABLE 1: Mean score (range) of overall and overlapping individual OSATS and GOALS domains}

OSATS: Objective Structured Assessment of Technical Skills

GOALS: Global Operative Assessment of Laparoscopic Skills

A high correlation was demonstrated between overall OSATS and GOALS assessments with a Pearson's correlation coefficient of $0.96(\mathrm{p}=0.01)$ (Figure 4).

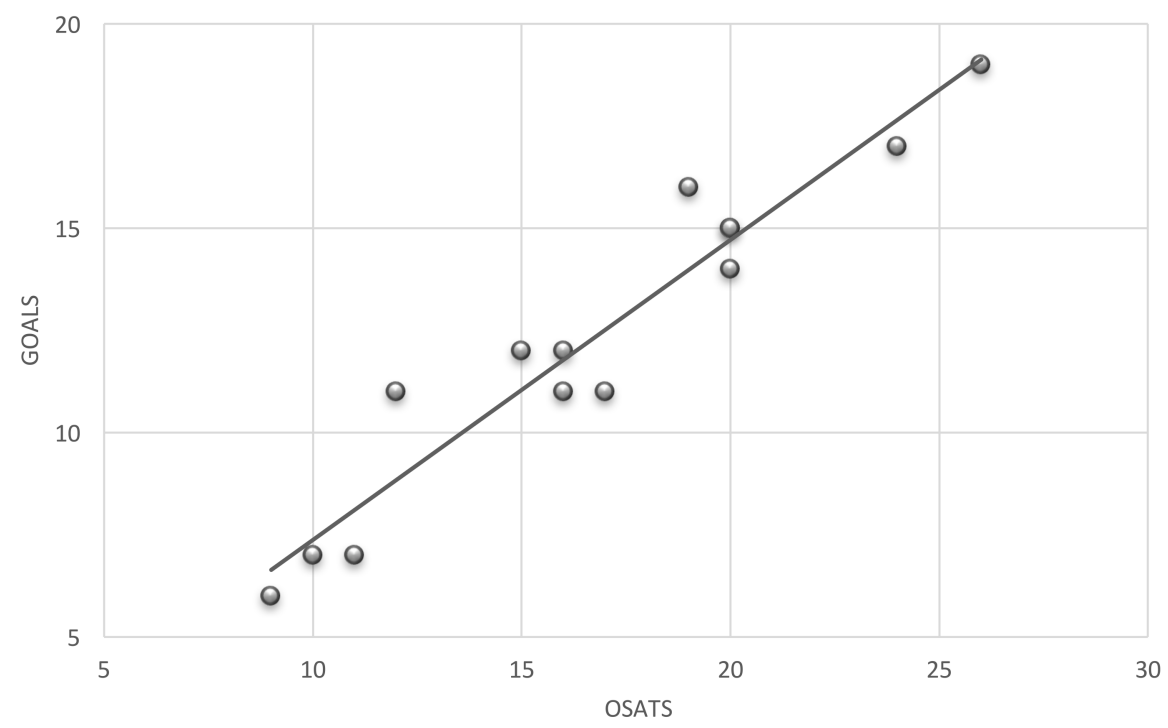

FIGURE 4: Pearson's correlation coefficient between participants OSATS and GOALS scores, R2=0.92

Note : 2 of 14 participants had identical scores and are seen only as one data point (OSATS $=20$, GOALS $=$ 15)

OSATS: Objective Structured Assessment of Technical Skills

GOALS: Global Operative Assessment of Laparoscopic Skills

Each overlapping domain also demonstrated high correlation (range $0.78-0.89, \mathrm{p}<0.05$, Table 2.) 


\section{Cureus}

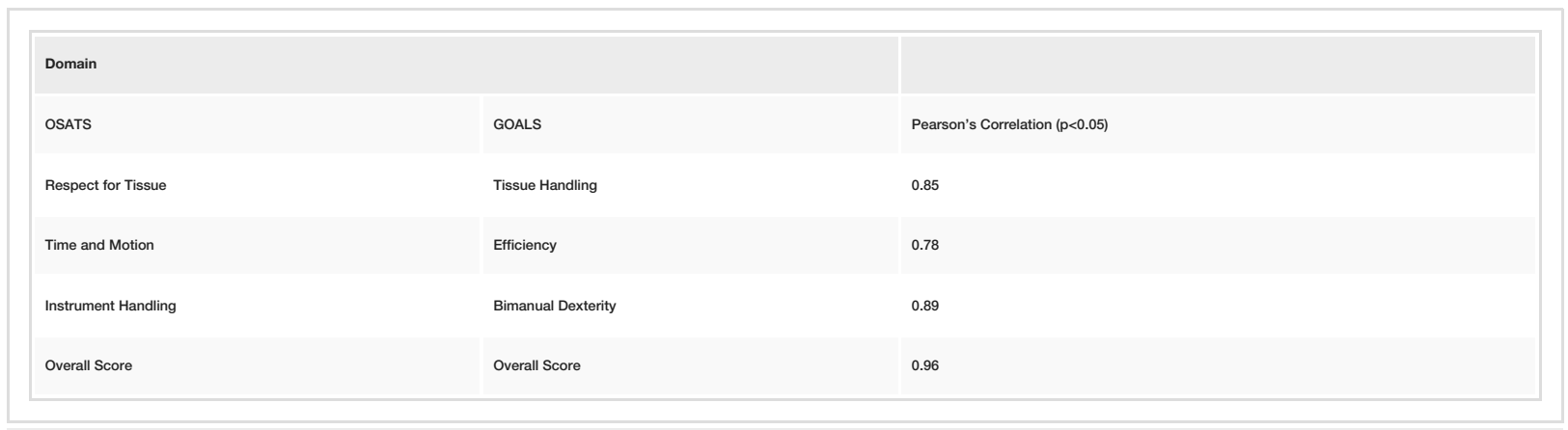

TABLE 2: Pearson's Correlation for overlapping OSATS and GOALS domains

OSATS: Objective Structured Assessment of Technical Skills

GOALS: Global Operative Assessment of Laparoscopic Skills

\section{Discussion}

OSATS and GOALS have been shown to be valid and reliable assessment tools for bench and operative settings in multiple investigations internationally and within our own institution [6-7, 13-14, 16-17]. In this investigation, concurrent evidence for validity was demonstrated by showing a near total correlation between the two scales' overall scores and in their overlapping domains. A strength of this protocol is that the porcine model allowed trainees to engage in a high-fidelity in vivo operative model without attendingsurgeon intervention, as may be required for safety in a human model. This allows a more accurate assessment of trainee skill, particularly in the novice cohort.

Essentially, Pearson's correlation is a measure of overlap between different scales. High values imply that scales are measuring similar constructs, while low values imply that scales are measuring dissimilar ones. The high correlation found suggests that OSATS and GOALS assess similar domains.

This finding questions the need for a separate laparoscopic assessment form as the more general OSATS may be used as effectively for assessment of laparoscopic skills. Advantages of using a single form for assessment in surgical education are many. First, it provides a common framework for use by raters. This may allow for a more consistent nomenclature, thus standardizing assessments across surgical platforms. This may improve reliability. Second, it is not limited by surgical approaches or platforms. A single scale may be applied to open, laparoscopic, laparoscopic-assisted, laparoscopic-converted-to-open, and potentially combined laparoscopic-endoscopic procedures.

These advantages may tempt one to conclude that is better for programs to focus on OSATS as it is validated for multiple general technical skills. However, this inference does not account for other valuable aspects of trainee assessment. Namely, it does not address the importance of feedback. While it is apparent that both forms have domains that grossly overlap, the correlation does not account for performance inferences learners may glean from the more specific assessment method. For example, the GOALS domain of bimanual dexterity significantly correlates with the OSATS domain of instrument handling $(\mathrm{R}=0.89)$. However, the specific anchor points on GOALS for this domain (e.g., $3=$ "uses both hands, but does not optimize interaction between hands") may provide more specific feedback to the learner in laparoscopic surgery than the more general OSATS assessment (e.g., $3=$ "competent use of instruments but occasionally appears stiff or awkward”). Thus, both tools are acceptable in making summative assessments of laparoscopic skills as they appear to measure an overarching competency-set. However, further investigation is needed to determine the degree to which technique-specific assessments afford formative feedback in the learning process.

We believe the results of this preliminary study warrants further investigation into the use of general and technique-specific assessments for evaluating laparoscopic skills in surgical education. However, this study does have limitations. These limitations include the results being drawn from a single institution with only 14 participants and using a limited number of raters. Although this number is consistent with related educational, preliminary studies, each form has known a high inter-rater agreement. A multicenter study with a larger sample size would strengthen the results.

\section{Conclusions}

General and technique-specific assessments of laparoscopic skill appear to map similar domains and are shown to correlate highly. This correlation has implications for their use in both summative and formative contexts. 


\section{Additional Information \\ Disclosures}

Human subjects: Consent was obtained by all participants in this study. University of Manitoba Health Research Ethics Board issued approval HS13026. Animal subjects: University of Manitoba Bannatyne Campus Animal Protocol Management and Review Committee Issued protocol number 15-020. Conflicts of interest: In compliance with the ICMJE uniform disclosure form, all authors declare the following: Payment/services info: All authors have declared that no financial support was received from any organization for the submitted work. Financial relationships: All authors have declared that they have no financial relationships at present or within the previous three years with any organizations that might have an interest in the submitted work. Other relationships: All authors have declared that there are no other relationships or activities that could appear to have influenced the submitted work.

\section{References}

1. Rivard JD, Vergis AS, Unger BJ, et al.: Construct validity of individual and summary performance metrics associated with a computer-based laparoscopic simulator. Surg Endosc. 2014, 28:1921-28. 10.1007/s00464013-3414-5

2. CanMEDS Framework. (2017). Accessed: July 29, 2017: http://canmeds.royalcollege.ca/en/framework.

3. Feldman LS, Sherman V, Fried GM: Using simulators to assess laparoscopic competence: ready for widespread use?. Surgery. 2004, 135:28-42. 10.1016/S0039-6060(03)00155-7

4. Turnbull J, Gray J, MacFadyen J: Improving in-training evaluation programs. J Gen Intern Med. 1998, 13:317-23. 10.1046/j.1525-1497.1998.00097.x

5. Fried GM, Feldman LS.: Objective assessment of technical performance. World J Surg. 2008, 32:156-60. 10.1007/s00268-007-9143-y

6. Martin JA, Regehr G, Reznick R, et al.: Objective structured assessment of technical skill (OSATS) for surgical residents. Br J Surg. 1997, 84:273-78. 10.1046/j.1365-2168.1997.02502.x

7. Reznick R, Regehr G, MacRae H, et al.: Testing technical skill via an innovative "bench station" examination . Am J Surg. 1997, 173:226-30. 10.1016/S0002-9610(97)89597-9

8. Van Hove PD, Tuijthof GJ, Verdaasdonk EG, et al.: Objective assessment of technical surgical skills. Br J Surg. 2010, 97:972-87. 10.1002/bjs.7115

9. Larsen CR, Grantcharov T, Schouenborg L, et al.: Objective assessment of surgical competence in gynaecological laparoscopy: development and validation of a procedure-specific rating scale. BJOG. 2008, 115:908-16. 10.1111/j.1471-0528.2008.01732.x

10. Aggarwal R, Grantcharov T, Moorthy K, et al.: An evaluation of the feasibility, validity, and reliability of laparoscopic skills assessment in the operating room. Ann Surg. 2007, 245:992-99. 10.1097/01.sla.0000262780.17950.e5

11. Scott DJ, Bergen PC, Rege RV, et al.: Laparoscopic training on bench models: better and more cost effective than operating room experience?. J Am Coll Surg. 2000, 191:272-83. 10.1016/S1072-7515(00)00339-2

12. Scott DJ, Rege RV, Bergen PC, et al.: Measuring operative performance after laparoscopic skills training: edited videotape versus direct observation. J Laparoendosc Adv Surg Tech A. 2000, 10:183-90. $10.1089 / 109264200421559$

13. Vassiliou MC, Feldman LS, Andrew CG, et al.: A global assessment tool for evaluation of intraoperative laparoscopic skills. Am J Surg. 2005, 107-13. 10.1016/j.amjsurg.2005.04.004

14. Gumbs AA, Hogle NJ, Fowler DL: Evaluation of resident laparoscopic performance using global operative assessment of laparoscopic skills. J Am Coll Surg. 2007, 308-13. 10.1016/j.jamcollsurg.2006.11.010

15. MacRae H, Regehr G, Leadbetter W, Reznick RK : A comprehensive examination for senior surgical residents . Am J Surg. 2000, 179:190-93. 10.1016/S0002-9610(00)00304-4

16. Steigerwald S, Park J, Hardy K, et al.: Does laparoscopic simulation predict intraoperative performance? A comparison between the Fundamentals of Laparoscopic Surgery and LapVR evaluation metrics. Am J Surg. 2015, 209:34-39. 10.1016/j.amjsurg.2014.08.031

17. Niitsu H, Hirabayashi N, Yoshimitsu M, et al.: Using the Objective Structured Assessment of Technical Skills (OSATS) global rating scale to evaluate the skills of surgical trainees in the operating room. Surg Today. 2013, 43:271-75. 10.1007/s00595-012-0313-7 